\title{
Campylobacter jejuni Induces Colitis through Activation of Mammalian Target of Rapamycin Signaling
}

\author{
Xiaolun Sun ${ }^{1}$, Deborah Threadgill ${ }^{2}$, and Christian Jobin ${ }^{1,3,4}$ \\ ${ }^{1}$ Department of Medicine, University of North Carolina, Chapel Hill, NC \\ ${ }^{3}$ Department of Pharmacology, University of North Carolina, Chapel Hill, NC \\ ${ }^{4}$ Department of Microbiology and Immunology, University of North Carolina, Chapel Hill, NC \\ ${ }^{2}$ Department of Microbiology, North Carolina State University, Raleigh, NC
}

\begin{abstract}
BACKGROUND \& AIMS-Campylobacter jejuni is the worldwide leading cause of bacterialinduced enteritis. The molecular and cellular events that lead to campylobacteriosis are poorly understood. We identify mammalian target of rapamycin (mTOR) as a signaling pathway that leads to $C$ jejuni-induced intestinal inflammation.
\end{abstract}

METHODS-Germ-free (control) or conventionally-derived interleukin (Il)10-/- mice that express enhanced green fluorescent protein under the control of NF- $\kappa \mathrm{B}\left(I l 10-/-; \mathrm{NF}-\mathrm{KB}^{\mathrm{EGFP}}\right.$ mice) were infected with $C$ jejuni (109 CFU/mouse) for 12 days; their responses were determined using histologic, semi-quantitative reverse transcription PCR, fluorescence in situ hybridization, transmission electron microscopy, and tissue culture analyses. mTOR signaling was blocked by daily intraperitoneal injections of the pharmacologic inhibitor rapamycin $(1.5 \mathrm{mg} / \mathrm{kg}) . \mathrm{CD} 4^{+} \mathrm{T}$ cells were depleted by intraperitoneal injections of antibodies against CD4 $(0.5 \mathrm{mg} / \mathrm{mouse}$, every 3 days). Bacterial survival in splenocytes was measured using a gentamycin killing assay.

RESULTS- $C$ jejuni induced intestinal inflammation, which correlated with activation of mTOR signaling and neutrophil infiltration. The inflamed intestines of these mice had increased levels of Il-1 $\beta$, Cxcl2, Il-17a, and EGFP; $C$ jejuni localized to colons and extra-intestinal tissues of infected Il10-I-; $N F-\kappa B^{E G F P}$ mice, compared with controls. Rapamycin, administered before or after introduction of $C$ jejuni, blocked $C$ jejuni-induced intestinal inflammation and bacterial accumulation. LC3II processing and killing of $C$ jejuni were increased in splenocytes incubated with rapamycin, compared with controls.

CONCLUSIONS-mTOR signaling mediates $C$ jejuni-induced colitis in $I l 10-/-$ mice, independently of T-cell activation. Factors involved in mTOR signaling might be therapeutic targets for campylobacteriosis.

(C) 2011 The American Gastroenterological Association. Published by Elsevier Inc. Allrights reserved

Correspondence: Christian Jobin, Ph.D. University of North Carolina Chapel Hill, NC 27599 USA Phone: +1-919-966-7884 Fax: +1-919-966-7468 job@med.unc.edu.

Publisher's Disclaimer: This is a PDF file of an unedited manuscript that has been accepted for publication. As a service to our customers we are providing this early version of the manuscript. The manuscript will undergo copyediting, typesetting, and review of the resulting proof before it is published in its final citable form. Please note that during the production process errors may be discovered which could affect the content, and all legal disclaimers that apply to the journal pertain.

Disclosures: The authors have declared that no competing interests exist.

Author Contribution: Conceived and designed the experiments: CJ, XS. Performed the experiments: XS. Analyzed the data: XS, CJ.

Contributed reagents: DT. Wrote the paper: XS, CJ. 


\section{Keywords}

crypt abscesses; imaging; mouse model; immune response; signal transduction

\section{INTRODUCTION}

Campylobacter jejuni is a gram-negative category B priority food- and water-borne pathogen and the worldwide leading bacterial causative agent of enteritis. ${ }^{1}$ The Centers for Disease Control and Prevention estimate that 2.4 million subjects are infected with $C$. jejuni resulting in 124 deaths every year in the United States. ${ }^{2}$ ENREF 2 ENREF 2 Clinical symptoms of $C$. jejuni infection include abdominal cramps, watery to bloody diarrhea, fever and gastrointestinal inflammation. ${ }^{3}$ At the cellular level, $C$. jejuni infected patients display infiltration of immune cells such as neutrophils, crypt abscesses and presence of fecal leukocytes. ${ }^{4}$ Although the intestinal disease self-resolves within one week, a small portion of patients (1:1000) develop extra-intestinal sequelae such as Guillain-Barre Syndrome (GBS) and reactive arthritis. ${ }^{5}$ Interestingly, $C$. jejuni exposure is also responsible for initiation and relapse of inflammatory bowel diseases (IBD) ${ }^{6}$ and post-infectious irritable bowel syndrome ${ }^{7}$.

Despite the prevalence of $C$. jejuni induced illness and negative socio-economic impact, a paucity of information is available regarding the molecular and cellular events involved in campylobacteriosis. The major contributing factor to our poor understanding of campylobacteriosis is the lack of robust experimental models mimicking the various phases of acute human infection. Although some mammals including monkey, ${ }^{8}$ ferret $^{9}$ and piglet ${ }^{10}$ have provided valuable information regarding cellular events associated with campylobacteriosis, limited reagents and lack of genetic manipulation in these models have constrained the generation of deep mechanistic understanding. We recently established an acute model of campylobacteriosis using germ-free $I l 10^{-1-}$ mice. ${ }^{11}$ In this model, $C$. jejuni induces a rapid (5 days) and robust inflammatory (bloody diarrhea) response to the microorganism. However, the cellular and molecular details responsible for this host response remained undefined.

Mammalian target of rapamycin (mTOR) plays an important role in cell growth and proliferation. ${ }^{12}$ The mTOR complex is composed of two entities: mTOR complex 1 (mTORC1) and mTOR complex 2 (mTORC2). mTORC1 is a downstream target of phosphoinositide 3-kinases (PI3K) and is sensitive to the pharmacological inhibitor rapamycin. ${ }^{13}$ The PI3K/mTOR pathway has long been implicated in regulating adaptive immunity by targeting $\mathrm{T}$ cell proliferation and activation. ${ }^{14}$ Recent evidence implicates PI3K/mTOR signaling as a key regulatory pathway of the innate immune host response. For example, rapamycin suppresses GM-CSF or IL-8 induced human neutrophil migration in vitro. ${ }^{15}$ In addition, PI3K signaling mediates IL-17 expression in inflamed murine lungs. ${ }^{16}$ Interestingly, IL-17 drives early neutrophil infiltration induced by i.p. Escherichia coli infection in mice. ${ }^{17}$ Finally, mTOR signaling modulates autophagic responses involved in bacterial killing and clearance. ${ }^{18}$

In this study, we hypothesized that mTOR represents an important signaling event regulating host response to $C$. jejuni infection. Our findings demonstrated that mTOR signaling regulates $C$. jejuni induced expression of inflammatory mediators, neutrophil infiltration and bacterial clearance. These molecular and signaling events represent critical steps in $C$. jejuni induced intestinal inflammation and could define potential new therapeutic targets. 


\section{MATERIALS AND METHODS}

\section{Mice}

All animal protocols were approved by the Institutional Animal Care and Use Committee of the University of North Carolina at Chapel Hill. Germ-free 8-12 week old $I l 10^{-/-}$; NF$\kappa B^{\mathrm{EGFP}}(129 / \mathrm{SvEv}$; $\mathrm{C} 57 \mathrm{BL} / 6$ mixed background) mice were transferred from germ-free isolators and immediately gavaged with $10^{9} C$. jejuni cfu/mouse (strain 81-176 ${ }^{19}$ ). Mice were then housed under Specific Pathogen-Free (SPF) conditions and sacrificed at 4 or 12 days. Germ-free $1110^{-1-} ; \mathrm{NF}-\kappa^{\mathrm{EGFP}}$ mice were also gavaged with $10^{7}$ Salmonella Typhimurium (S. Typhimurium strain CA32) cfu/mouse for 2 days or $10^{7}$ Escherichia coli (E. coli strain NC 101) cfu/mouse for 12 days. Conventionally-derived $\mathrm{IllO}^{-1-}$; NF$\kappa B^{\mathrm{EGFP}}$ mice were put on an antibiotic cocktail (streptomycin $2 \mathrm{~g} / \mathrm{L}$, gentamycin $0.5 \mathrm{~g} / \mathrm{L}$, bacteriocin $1 \mathrm{~g} / \mathrm{L}$ and ciprofloxacin $0.125 \mathrm{~g} / \mathrm{L}$ ) in water for 7 days and antibiotics were withdrawn $24 \mathrm{hr}$ prior infection. The mice were then gavaged with $10^{9} \mathrm{C}$. jejuni cfu/mouse for 14 days. To inhibit mTOR signaling, mice were injected daily i.p. with rapamycin (1.5 $\mathrm{mg} / \mathrm{kg}$ ) (Fisher Scientific). At the end of the experiment, mice were euthanized using $\mathrm{CO}_{2}$ intoxication. Colon, mesenteric lymph nodes (MLN) and spleen were collected for further processing RNA, protein or $C$. jejuni culture on Campylobacter selective blood plates (Remel) for $48 \mathrm{~h}$ at $37{ }^{\circ} \mathrm{C}$ using the GasPak system (BD). Colons were fixed in $10 \%$ buffered formalin (Fisher Scientific) overnight, paraffin-embedded, sectioned, and stained with H\&E for histological evaluation. Images were acquired using a DP71 camera and DP Controller 3.1.1.276 (Olympus). Intestinal inflammation was scored by evaluating the degree of lamina propria immune cell infiltration, goblet cell depletion, architectural distortion, as well as crypt hyperplasia, ulceration, and abscesses using a score from $0-4 .{ }^{11}$ Cecal and colonic inflammation induced by $S$. Typhimurium was evaluated using a score from $0-13$ as described. ${ }^{20}$

\section{CD4+ cell depletion}

C. jejuni infected mice were injected with anti-CD4 antibody (BioXcell) (i.p. $0.5 \mathrm{mg} / \mathrm{mouse}$, every 3 days) to deplete CD4+ cells. At the end of the experiment, mice were euthanized using $\mathrm{CO}_{2}$ intoxication. Colons were resected and processed for $\mathrm{H} \& \mathrm{E}$ staining and colitis evaluation. Spleen and MLN were collected and processed into single cell suspensions after erythrocyte lysis.

\section{Western blotting}

Whole tissues were lysed in Laemmli buffer and $20 \mu \mathrm{g}$ of protein was separated by SDSPAGE, transferred to nitrocellulose membranes and protein detected using enhanced chemiluminescence reaction (ECL) as described previously. ${ }^{11}$ Primary antibodies used were phospho-p70S6K (T389) and p70S6K (Cell Signaling), EGFP (Sigma), LC3 I/II (MBL) and Actin (BP Biomedicals).

\section{Enhanced GFP (EGFP) macro- and micro-imaging}

$I l 10^{-/-}$; NF- $\mathrm{KB}^{\mathrm{EGFP}}$ mice were sacrificed; the colon was dissected and immediately imaged using a charge-coupled device camera in a light-tight imaging box with a dual-filtered light source and emission filters specific for EGFP (LT-99D2 Illumatools; Lightools Research). For microimaging, colonic segments were cut open and fixed in $4 \%$ paraformaldehyde overnight at $4{ }^{\circ} \mathrm{C}$, and then permeabilized in $2 \%$ triton overnight at $4^{\circ} \mathrm{C}$. The tissues were then immersed in FocusClear (CelExplorer labs) and then stained with DAPI. The tissues were put on slides with lumen side facing the lens. NF- $\mathrm{kB}$ derived EGPF were examined using Olympus FV1000 confocal multiphoton upright microscope system with Olympus 
Fluoview 2.0 software. Acquired images were reconstructed into 3-D using Imaris (Bitplane).

\section{Fluorescence in situ hybridization (FISH)}

Cy3-tagged 5'AGCTAACCACACCTTATACCG3 ${ }^{121}$ was used to probe the presence of $C$. jejuni. Deparaffinized, formalin-fixed $5 \mu \mathrm{m}$ thick sections were incubated for 15 minutes in lysozyme (300,000 Units/ml lysozyme; Sigma-Aldrich) buffer ( $25 \mathrm{mM}$ Tris $\mathrm{pH} 7.5,10 \mathrm{mM}$ EDTA, $585 \mathrm{mM}$ sucrose, and $0.3 \mathrm{mg} / \mathrm{ml}$ sodium taurocholate) at room temperature and hybridized overnight at $46^{\circ} \mathrm{C}$ in hybridization chambers with the oligonucleotide probe (final concentration of $5 \mathrm{ng} / \mu \mathrm{l}$ in a solution of 30 percent formamide, $0.9 \mathrm{M}$ sodium chloride, $20 \mathrm{mM}$ Tris $\mathrm{pH} 7.5$, and $0.01 \%$ sodium dodecyl sulfate). Tissue sections were washed for 20 minutes at $48{ }^{\circ} \mathrm{C}$ in washing buffer $(0.9 \mathrm{M}$ sodium chloride, $20 \mathrm{mM}$ Tris $\mathrm{pH}$ 7.5) and once in distilled water for 10 seconds, dried at $46^{\circ} \mathrm{C}$, mounted with DAPI mount media, and imaged using a Zeiss LSM710 Spectral Confocal Laser Scanning Microscope system with ZEN 2008 software. Acquired images were analyzed using BioimageXD. ${ }^{22}$ For whole tissue FISH, colons were fixed in $4 \%$ paraformaldehyde overnight at $4{ }^{\circ} \mathrm{C}, 2 \%$ triton overnight at $4{ }^{\circ} \mathrm{C}$, and $C$. jejuni probe was added and incubated overnight at $46^{\circ} \mathrm{C}$. The whole tissue was immersed in FocusClear, imaged on an Olympus FV1000 microscope and reconstructed into 3-D using Imaris.

\section{Immunohistochemistry (IHC)}

Deparaffinized colon sections were treated with $3 \% \mathrm{H}_{2} \mathrm{O}_{2}$ for 10 minutes to quench endogenous peroxidases. Antigen retrieval was performed using microwave heating for 10 $\min$ in $10 \mathrm{mM}$ citrate buffer $\mathrm{pH}$ 6.0. Sections were blocked in 5\% goat serum TBS-T for 1 hour. The slides were incubated overnight at $4^{\circ} \mathrm{C}$ with an anti-MPO antibody $(1: 400)$ (Thermo Scientific). Secondary biotinylated antibody was diluted at 1:1000 with the Vectastain ABC Elite Kit (Vector Laboratories). Visualization was performed using DAB chromogen (DAKO). Finally, sections were counterstained with hematoxylin for 5 seconds. Sections were imaged at the Olympus microscope using DP 71 camera and DP Controller 3.1.1.276 (Olympus).

\section{Statistical analysis}

Values are shown as mean \pm SEM as indicated. Differences between groups were evaluated with the nonparametric Mann-Whitney U test. Experiments were considered statistically significant if $P$ values $<0.05$. All calculations were performed using Prim 5.0 software.

\section{RESULTS}

\section{mTOR mediates $C$. jejuni-induced colitis}

Although the role of mTOR has moved beyond that of a modulator of T cell function, the involvement of this multifunctional kinase in host responses to pathogenic bacteria has not been clearly explored. To investigate the impact of mTOR on $C$. jejuni-induced colitis in vivo, germ-free $I l 10^{-/-}$; NF- $\mathrm{\kappa B}^{\mathrm{EGFP}}$ mice were transferred to specific pathogen free (SPF) housing and immediately gavaged with $C$. jejuni $\left(10^{9} \mathrm{CFU} /\right.$ mouse $)$ and then injected i.p. daily with either vehicle (5\% DMSO PBS) or rapamycin ( $1.5 \mathrm{mg} / \mathrm{kg}$ body weight) for 12 days. Western blot analysis showed that rapamycin attenuated $C$. jejuni induced phosphorylation of p70S6 kinase (T389), a downstream target of mTOR (Fig. 1A). As previously reported, ${ }^{11}{ }^{I l l O^{-/-}}$; NF- $\mathrm{KB}^{\mathrm{EGFP}}$ mice infected with $C$. jejuni showed severe intestinal inflammation as seen by extensive immune cell infiltration, epithelial ulceration, goblet cell depletion and crypt hyperplasia and abscesses compared to uninfected mice (Fig. 1B and 1D). Interestingly, rapamycin blocked $C$. jejuni-induced intestinal inflammation in 
$1 l 10^{-/-} ; \mathrm{NF}-\mathrm{\kappa B}^{\mathrm{EGFP}}$ mice. Since germ free mice have an immature immune system, we next treated conventionally-derived $I l 10^{-1-} ; \mathrm{NF}-\kappa^{\mathrm{EGFP}}$ mice with antibiotics and then infected these mice with $C$. jejuni for 12 days. As observed in germ free mice, $C$. jejuni induced severe intestinal inflammation in conventionally-derived $I_{110^{-1-}}$; NF- $\mathrm{KB}^{\mathrm{EGFP}}$ mice, an effect significantly attenuated by rapamycin exposure (Supplemental Fig. S1a-b).

To determine the specificity of rapamycin on other colitogenic microorganisms, we infected germ free $I l 10^{-1-}$; NF- $\mathrm{KB}^{\mathrm{EGFP}}$ mice with the enteric pathogens Salmonella Typhimurium ( $10^{7} \mathrm{CFU} /$ mouse) for 2 days or Escherichia coli NC 101 (10 $0^{7} \mathrm{CFU} /$ mouse) for 12 days. Interestingly, E. coli infected $I l 10^{-/-}$; NF-кB ${ }^{\text {EGFP }}$ mice did not develop intestinal inflammation at this time point, suggesting a specific host response to C.jejuni infection (Supplemental Fig. S2a-b). However, $I l 10^{-/-}$; NF- $\mathrm{kB}^{\mathrm{EGFP}}$ mice infected with $S$. Typhimurium developed severe cecal and colonic inflammation, which was strongly attenuated by rapamycin exposure (Supplemental Fig. S3). These results suggest that mTOR signaling mediates deleterious responses from different enteropathogenic microorganisms.

C. jejuni induced intestinal inflammation is evident as early as day 4 in $I l 10^{-1-}$; NF- $\mathrm{KB}^{\mathrm{EGFP}}$ infected mice (Supplemental Fig. S4a-b). ${ }^{11}$ To further test if rapamycin may be used as an intervention agent, we injected the inhibitor daily to C. jejuni infected (4 days) $I l 10^{-/-}$; NF$\kappa B^{\mathrm{EGFP}}$ mice and collected intestinal tissues at 12 days. Remarkably, rapamycin treatment strongly reversed $C$. jejuni induced intestinal inflammation and bloody diarrhea (Fig. 1C and 1E, Supplemental Fig. S5a-b).

Since rapamycin has immunomodulatory effects on $\mathrm{T}$ cells, we depleted CD4+ T cells using an anti-CD4 antibody ( $0.5 \mathrm{mg} / \mathrm{mouse} / \mathrm{every}$ three days) to address the function of these cells in the acute phase of campylobacteriosis. Notably, $C$. jejuni induced colitis was only slightly inhibited ( 20\%) in CD4+ T cell-depleted mice compared to control (Fig. 2A-B). Complete depletion of CD4 cells was observed in the spleen and MLN cellular compartment of antibody-treated mice (Fig. 2C-D). These results suggest that the early phase of $C$. jejuniinduced colitis is mostly mediated by an innate immune response and that rapamycin likely targets activities of innate immune cells.

\section{mTOR regulates $C$. jejuni induced NF-kB activity and proinflammatory gene expression}

We previously showed that NF- $\mathrm{\kappa B}$ is activated in $C$. jejuni infected $I l 10^{-1-}$; NF- $\mathrm{KB}^{\mathrm{EGFP}}$ mice. ${ }^{11}$ To evaluate the impact of mTOR signaling on NF-kB activity, EGFP expression in the colon of $I l 10^{-1-}$; NF- $\mathrm{KB}^{\mathrm{EGFP}}$ mice was visualized using a $\mathrm{CCD}$ camera macroimaging system. $I l 10^{-1-}$; NF-kB ${ }^{\text {EGFP }}$ mice infected with $C$. jejuni displayed enhanced colonic NF$\kappa \mathrm{B}^{\mathrm{EGFP}}$ expression with strongly positive lymphoid aggregates (arrow heads) compared to uninfected mice (Fig. 3A). Interestingly, the colon of rapamycin-treated, C. jejuni-infected $I l 10^{-1-}$; NF- $\mathrm{KB}^{\mathrm{EGFP}}$ mice displayed reduced EGFP expression compared to untreated, infected-mice. Consistent with these findings, western blot analysis of colonic lysates demonstrated reduced EGFP expression in rapamycin-treated, $C$. jejuni-infected mice (Fig. 3B).

To further evaluate the distribution of NF- $\mathrm{KB}^{\mathrm{EGFP}}$ positive cells within the colon, whole colonic tissue was visualized using confocal microscopy coupled with 3-D image reconstruction. In accordance with the macroimaging and Western blot data, C. jejuniinfected $I l 10^{-1-}$; NF- $\mathrm{kB}^{\mathrm{EGFP}}$ mice showed numerous EGFP positive cells in the colonic lamina propria compared to uninfected mice (Fig. 3C and supplementary Fig3video1.mov, Fig3video2.mov, Fig3video3.mov and Fig3video4.mov). Strikingly, colonic EGFP tissue expression was strongly reduced in rapamycin-treated, C. jejuni-infected $\mathrm{IllO}^{-1-}$; NF$\kappa \mathrm{B}^{\mathrm{EGFP}}$ mice compared to untreated mice. 
We next examined the impact of rapamycin on expression of various NF- $\kappa \mathrm{B}$ dependent proinflammatory mediators involved in bacterial host responses. $C$. jejuni infection strongly induced $I 1-1 \beta, C x c l 2$ and $I l-17 a$ mRNA accumulation in colonic tissue of $1110^{-1-}$; NF$\mathrm{\kappa B}^{\mathrm{EGFP}}$ mice, effects attenuated by 79,75 , and $92 \%$, respectively in rapamycin-treated, $C$. jejuni-infected mice (Fig. 4A). Consistent with these findings, secreted IL-1 $\beta$ and IL-17 from supernatant of cultured colon explants was reduced by $59 \%$ and $94 \%$ respectively while production of these cytokines was attenuated by $41 \%$ and $82 \%$ in MLN of rapamycintreated, $C$. jejuni-infected mice compared to control mice (Fig. 4B). Interestingly, $C$. jejuniinduced colonic $I l-12 p 40$ and Tnfa mRNA accumulation were not significantly inhibited by rapamycin treatment, suggesting that inflammatory mediators are selectively affected by mTOR signaling (Supplemental Fig. S6).

\section{Neutrophils participate in $C$. jejuni-induced colitis}

Neutrophil infiltration is a hallmark of acute infection and represents an important feature of human campylobacteriosis. ${ }^{4}$ Of note, rapamycin strongly reduced $C$. jejuni induced expression of the neutrophil marker myeloperoxidase (MPO) in colonic tissue of infected $I l 10^{-1-}$; NF-kB ${ }^{\mathrm{EGFP}}$ mice (Fig. $5 \mathrm{~A}$ ). Moreover, the ratio of $\mathrm{Gr}-1^{+}: \mathrm{CD} 45^{+}$cells significantly decreased $(41 \%)$ in the blood of rapacymin-treated, $C$. jejuni infected mice compared to untreated, infected mice (Fig. 5B). Finally, transmission electron microscope (TEM) analysis showed neutrophil accumulation in colonic crypt, which associates with microvilli/ glycocalyx destruction (Fig. 5C). Notably, these pathological features were not observed in rapamycin-treated mice (Fig. 5C, right panel).

\section{mTOR mediates $C$. jejuni invasion}

We next investigated the impact of mTOR signaling on intestinal and extra-intestinal $C$. jejuni tissue distribution. Following infection and treatment with rapamycin, $C$. jejuni DNA was visualized in the colon of $I l 10^{-1-}$; NF- $\mathrm{KB}^{\text {EGFP }}$ mice using fluorescence in situ hybridization (FISH) and confocal microscopy imaging. Interestingly, while $C$. jejuni was detected deeply in the inflamed crypts and in the lamina propria section of the intestine of untreated mice, $C$. jejuni DNA was barely detectable in rapamycin-treated mice (Fig. 6A).

To gain a better perspective of $C$. jejuni invasion, FISH was performed using fresh-fixed colonic tissues sections, and then imaged with confocal microscopy coupled with 3-D visualization. Interestingly, while $C$. jejuni extensively invaded the colon of untreated mice, its presence is strongly reduced in rapamycin-treated mice (Fig. 6B and supplementary Fig6video1.mov and Fig6video2.mov). To further characterize $C$. jejuni invasion, colonic tissue was also imaged using TEM. The presence of spirally shaped $C$. jejuni (arrow) lacking its outer membrane was detected in epithelial cells (Fig. 6C left panel). Also the absence of a vesicular membrane around the invading bacteria suggests that phagosome formation in epithelial cells is not a host response to the bacterium. Consistent with FISH results, intracellular $C$. jejuni were virtually absent and microvilli were intact in epithelial cells of rapamycin-treated mice (Fig. $6 \mathrm{C}$ right panel).

Reduced $C$. jejuni in the colon of rapamycin-treated mice suggests that the bacteria were eliminated or were able to evade/translocate to extra-intestinal tissues. To resolve this issue, we aseptically collected samples from the stool, colon, spleen and MLN and enumerated $C$. jejuni on Remel Campylobacter selective plates. Consistent with the FISH and TEM results, rapamycin treatment reduced $C$. jejuni colonic tissue invasion by $90 \%$ compared to $C$. jejuni-infected, untreated mice (Fig. 6D). Furthermore, rapamycin treatment strongly reduced $C$. jejuni presence in the MLN and spleen of infected mice compared to untreated, infected mice. Altogether, these findings identified mTOR signaling and neutrophil infiltration as important events leading to $C$. jejuni invasion and pathogenesis. 
The above findings suggest that rapamycin favors bacterial clearance, which lead to decreased innate response and intestinal inflammation. To directly test the impact of rapamycin on bacterial survival, we infected splenocytes isolated from $I l 10^{-1-}$ mice with $C$. jejuni and performed a gentamycin killing assay. Interestingly, rapamycin enhanced $C$. jejuni killing in splenocytes at $4 \mathrm{hr}$ compared to untreated cell (Fig. 7A). Western blot analysis showed that rapamycin attenuated $C$. jejuni induced phosphorylation of p70S6 kinase (T389) and enhanced LC3 II conversion, a marker of autophagy (Fig.7B). Interestingly, $C$. jejuni exposed to rapamycin as high as $1 \mathrm{mM}$ did not alter the growth/ viability of the bacterium. Altogether, these findings showed that increased mTOR signaling following $C$. jejuni infection mediates proinflammatory response, likely by preventing hostmediated bacterial killing.

\section{DISCUSSION}

The fundamental molecular host response to $C$. jejuni infection remains virtually unknown. In this study, we present clear evidences that $C$. jejuni-mediated intestinal inflammation is caused by activation of the host mTOR signaling pathway and neutrophil infiltration. Comparable intestinal inflammation between CD4+ cells depleted and untreated mice indicates that $C$. jejuni induced colitis is mostly driven by innate immune cells at day 12 . Although CD4+ T cells are not involved in the development of intestinal inflammation by day 12 , adaptive host response is an important hallmark of campylobacteriosis. ${ }^{23}$ Interestingly and in contrast to $C$. jejuni, the adherent invasive $E$. coli $\mathrm{NC} 101$ failed to induce colitis after 12 days, whereas severe colitis developed after 10-20 weeks of colonization with the same bacterium. ${ }^{24}$ In addition, colitis was observed as early as two days in S. Typhimurium infected $I l 10^{-/-}$mice. These findings suggest a differential ability of bacteria to trigger intestinal inflammation in $I l 10^{-1-}$ mice. Using this robust and tractable animal model, we demonstrate that $C$. jejuni induced mTOR signaling leads to increased $\mathrm{NF}-\kappa \mathrm{B}$ activity and induction of NF- $\mathrm{kB}-$ dependent genes (cytokines/chemokines). FISH assay and electron microscopy analysis showed that $C$. jejuni rapidly invades the intestinal mucosal layer and triggers a host response involving neutrophil recruitment and formation of crypt ulcers. Disruption of the crypt architecture by massive neutrophil influx likely compromises the intestinal barrier integrity, which further favors bacterial uptake/invasion and dissemination to extra-intestinal tissues as seen by enhanced bacterial counts in the spleen and MLN. mTOR blockade with a pharmacological inhibitor strongly attenuated campylobacteriosis in $I l 10^{-1-}$ mice. Moreover, rapamycin was able to treat established $C$. jejuni-induced intestinal inflammation.

A close examination of human campylobacteriosis suggests a preponderant role of neutrophils in the course of the pathology. Histological assessment showed the presence of numerous crypt abscesses and neutrophil infiltration in the intestinal mucosa of infected patients. ${ }^{4}$ However, up until now the functional importance of neutrophil infiltration in $C$. jejuni-induced pathogenesis has not been investigated. Although the main biological function of neutrophils is to ingest and eliminate invading microorganisms, excessive infiltration of these innate immune cells and release of various degradative enzymes and oxidative products cause extensive collateral tissue damage to the host. For example, neutrophil accumulation causes elevated IL- $1 \beta$ and CXCL2 production and subsequent joint inflammation, an effect attenuated with anti-IL1 $\beta$ antibody treatment. ${ }^{25}$ Interestingly, TEM showed that the microvilli and associated glycocalyx of epithelial cells in neutrophilinfiltrated crypts are virtually ablated in $C$. jejuni-infected mice.-The molecular events leading to neutrophil infiltration in the intestine of $C$. jejuni-infected mice is not entirely clear. Induction of $\mathrm{Il}-17 \mathrm{a}$ and $\mathrm{Cxcl} 2$ genes is important for neutrophil expansion and recruitment. ${ }^{26,27}$ Interestingly, blocking mTOR with rapamycin decreased colonic $I l-17 a$ and $\mathrm{Cxcl} 2$ expression, which correlated with reduction of neutrophils infiltration and crypt 
abscesses and with disease improvement. Although IL-17 is a signature cytokine of the Th17 cells, our CD4 depletion experiment suggests that adaptive $\mathrm{CD}^{+} \mathrm{T}$ cells play a minor role in campylobacteriosis. IL-17 is produced by a wide array of innate immune cells including $\gamma \delta \mathrm{T}$ cells, natural killer $\gamma \delta \mathrm{T}$ cells and lymphoid tissue inducer cells. ${ }^{28}$ Since $\gamma \delta \mathrm{T}$ cells are highly abundant in the intestinal mucosa, it is tempting to speculate that these cells are the source of IL-17 in our model. Interestingly, in an E. coli infectious model, neutrophil recruitment to the peritoneal cavity is dependent on innate $\gamma \delta \mathrm{T}$ cell-mediated IL-17 secretion. ${ }^{17}$ Similarly, Klebsiella pneumonia-induced lung inflammation is attenuated in $\mathrm{Il}-17 r^{-1-}$ mice, which correlated with neutrophil recruitment and G-SCF and CXCL2 expression. ${ }^{27}$ Further studies would be needed to define the role of IL-17 in campylobacteriosis.

Another important function of mTOR signaling is the regulation of autophagy. Our findings indicate that $C$. jejuni infiltration in the colon, MLN and spleen is dramatically reduced in rapamycin-treated mice, suggesting increased bacterial killing. Using primary splenocytes, we found that rapamycin enhanced $C$. jejuni killing, which correlated with enhanced LC3II conversion. These findings suggest that $C$. jejuni induces mTOR signaling as a mean to avoid host-mediated killing, possibly by prevent adequate autophagy response. Further studies would be needed to definitively establish the role of autophagy in campylobacteriosis. Interestingly, S. Typhimurium induced colitis was also inhibited by rapamycin exposure, suggesting that mTOR may be the target of other enteropathogenic microorganisms. mTOR has also been recently identified as a negative regulator of LPSinduced NF- $\kappa \mathrm{B}$ signaling in monocytes, macrophage, and primary DC. ${ }^{29}$ Therefore, blocking mTOR signaling could enhance NF- $\mathrm{KB}$ signaling and production of inflammatory mediators involved in bacterial killing. However, our data do not support this hypothesis as EGFP expression (NF- $\kappa \mathrm{B}$ activity) and some NF- $\mathrm{\kappa B}$-dependent inflammatory mediators are reduced in rapamycin-treated mice. While we observed decreased activation of NF- $\mathrm{KB}$ (EGFP expression) and inflammatory gene expression in rapamycin-treated mice, these observations are likely a secondary effect of the drug since direct NF- $\mathrm{kB}$ inhibitors failed to prevent campylobacteriosis. ${ }^{11}$

Although the majority of $C$. jejuni infected patients recover within 2 to 10 days without any specific treatment, a significant proportion develop severe clinical symptoms (fever, bloody diarrhea and abdominal cramps) which requires antibiotic treatment to shorten symptom durance. ${ }^{1}$ Recently, there are increasing concerns over $C$. jejuni resistance to antibiotics ${ }^{30}$ and post-infectious sequelae such as GBS, IBD and IBS. ${ }^{5-7}$ Immune based prevention or intervention has attracted much attention as an alternative to antibiotic treatment. ${ }^{31}$ Based on our novel findings, specific mTOR inhibitors (such as Rapamune, Torisel, Afinitor and Zortress) already approved for human use could represent a potential alternative to antibiotic-based therapy to treat campylobacteriosis. Further work would be needed before mTOR is validated as a therapeutic target for campylobacteriosis.

In summary, this report defines the critical role of mTOR in mediating the pro-inflammatory effect of $C$. jejuni infection. We report that neutrophil infiltration plays an active role in pathogenesis of $C$. jejuni infection and modulation of the cellular/molecular events leading to this process could represent a new therapeutic arsenal to control campylobacteriosis.

\section{Supplementary Material}

Refer to Web version on PubMed Central for supplementary material. 


\section{Acknowledgments}

The authors would like to thank Dr. Sandy Kim and Ward Jarvis for guidance with the FISH assay. The authors would also like to thank Brigitte Allard for technical assistance throughout this project. We would also like to thank Dr. Robert Bagnell, Mr. Steven Ray and Mrs. Victoria Madden (Microscopy Services Laboratory) and Mr. Robert Currin (Manager, Cell \& Molecular Imaging Facility and UNC-Olympus Center), all of UNC-CH, for their assistance with the fluorescent microscopy, TEM experiments and 3-D reconstruction. Additionally, we would like to thank Dr. Fengling Li and Mrs. Maureen Bower (Gnotobiotic Core, UNC-CH) for performing C. jejuni gavage. Finally, we thank Dr. Carlton Anderson for assistance with ELISA assay (Immunology core, UNC-CH).

Funding: This research was supported by National Institutes of Health grants DK047700, DK073338, AI082319 to C. Jobin and by P30 DK34987 for the CGIBD. The funders had no role in study design, data collection and analysis, decision to publish, or preparation of the manuscript.

\section{Abbreviations}

mTOR mammalian target of rapamycin

MLN mesenteric lymph nodes

IHC immunohistochemistry

FISH Fluorescence in situ hybridization

TEM transmission electron microscope

ELISA Enzyme-linked immunosorbent assay

\section{References}

1. Allos BM. Campylobacter jejuni Infections: update on emerging issues and trends. Clin Infect Dis. 2001; 32:1201-6. [PubMed: 11283810]

2. CDC. [Accessed October 19, 2010] Campylobacter: General Information. 2010. Available at http://www.cdc.gov/nczved/divisions/dfbmd/diseases/campylobacter/.

3. Blaser MJ. Epidemiologic and clinical features of Campylobacter jejuni infections. J Infect Dis. 1997; 176(Suppl 2):S103-5. [PubMed: 9396691]

4. van Spreeuwel JP, Duursma GC, Meijer CJ, Bax R, Rosekrans PC, Lindeman J. Campylobacter colitis: histological immunohistochemical and ultrastructural findings. Gut. 1985; 26:945-951. [PubMed: 4029720]

5. Nachamkin I. Chronic effects of Campylobacter infection. Microbes Infect. 2002; 4:399-403. [PubMed: 11932190]

6. Gradel KO, Nielsen HL, Schønheyder HC, Ejlertsen T, Kristensen B, Nielsen H. Increased Shortand Long-Term Risk of Inflammatory Bowel Disease After Salmonella or Campylobacter Gastroenteritis. Gastroenterology. 2009; 137:495-501. [PubMed: 19361507]

7. Qin HY, Wu JC, Tong XD, Sung JJ, Xu HX, Bian ZX. Systematic review of animal models of postinfectious/post-inflammatory irritable bowel syndrome. J Gastroenterol. 2010 2010/09/18 ed. DOI: 10.1007/s00535-010-0321-6.

8. Russell RG, Blaser MJ, Sarmiento JI, Fox J. Experimental Campylobacter jejuni infection in Macaca nemestrina. Infect Immun. 1989; 57:1438-44. [PubMed: 2707853]

9. Nemelka KW, Brown AW, Wallace SM, Jones E, Asher LV, Pattarini D, Applebee L, Gilliland TC Jr. Guerry P, Baqar S. Immune response to and histopathology of Campylobacter jejuni infection in ferrets (Mustela putorius furo). Comp Med. 2009; 59:363-71. [PubMed: 19712577]

10. Law BF, Adriance SM, Joens LA. Comparison of in vitro virulence factors of Campylobacter jejuni to in vivo lesion production. Foodborne Pathog Dis. 2009; 6:377-85. [PubMed: 19278341]

11. Lippert E, Karrasch T, Sun X, Allard B, Herfarth HH, Threadgill D, Jobin C. Gnotobiotic IL-10; NF-kappaB mice develop rapid and severe colitis following Campylobacter jejuni infection. PLoS One. 2009; 4:e7413. [PubMed: 19841748] 
12. Wullschleger S, Loewith R, Hall MN. TOR signaling in growth and metabolism. Cell. 2006; 124:471-84. [PubMed: 16469695]

13. Inoki K, Li Y, Zhu T, Wu J, Guan K-L. TSC2 is phosphorylated and inhibited by Akt and suppresses mTOR signalling. Nature Cell Biology. 2002; 4:648-657.

14. Janes MR, Fruman DA. Immune Regulation by Rapamycin: Moving Beyond T Cells. Science Signaling. 2009; 2:pe25-pe25. [PubMed: 19383976]

15. Gomez-Cambronero J, Horn J, Paul CC, Baumann MA. Granulocyte-macrophage colonystimulating factor is a chemoattractant cytokine for human neutrophils: involvement of the ribosomal p70 S6 kinase signaling pathway. J Immunol. 2003; 171:6846-55. [PubMed: 14662891]

16. Kim SR, Lee KS, Park SJ, Min KH, Lee KY, Choe YH, Lee YR, Kim JS, Hong SJ, Lee YC. PTEN down-regulates IL-17 expression in a murine model of toluene diisocyanate-induced airway disease. J Immunol. 2007; 179:6820-9. [PubMed: 17982072]

17. Shibata K, Yamada H, Hara H, Kishihara K, Yoshikai Y. Resident Vdelta1+ gammadelta T cells control early infiltration of neutrophils after Escherichia coli infection via IL-17 production. J Immunol. 2007; 178:4466-72. [PubMed: 17372004]

18. Gutierrez MG, Master SS, Singh SB, Taylor GA, Colombo MI, Deretic V. Autophagy Is a Defense Mechanism Inhibiting BCG and Mycobacterium tuberculosis Survival in Infected Macrophages. Cell. 2004; 119:753-766. [PubMed: 15607973]

19. Korlath JA, Osterholm MT, Judy LA, Forfang JC, Robinson RA. A point-source outbreak of campylobacteriosis associated with consumption of raw milk. J Infect Dis. 1985; 152:592-6. [PubMed: 4031557]

20. Hapfelmeier S, Stecher B, Barthel M, Kremer M, Muller AJ, Heikenwalder M, Stallmach T, Hensel M, Pfeffer K, Akira S, Hardt WD. The Salmonella pathogenicity island (SPI)-2 and SPI-1 type III secretion systems allow Salmonella serovar typhimurium to trigger colitis via MyD88dependent and MyD88-independent mechanisms. J Immunol. 2005; 174:1675-85. [PubMed: 15661931]

21. Poppert S, Haas M, Yildiz T, Alter T, Bartel E, Fricke U, Essig A. Identification of Thermotolerant Campylobacter Species by Fluorescence In Situ Hybridization. Journal of Clinical Microbiology. 2008; 46:2133-2136. [PubMed: 18385439]

22. Kankaanpää P, Pahajoki K, Marjomäki V, Heino J, White D. BioImageXD-New Open Source Free Software for the Processing, Analysis and Visualization of Multidimensional Microscopic Images. Microscopy Today. 2006; 14:12-16.

23. Yuki N, Susuki K, Koga M, Nishimoto Y, Odaka M, Hirata K, Taguchi K, Miyatake T, Furukawa K, Kobata T, Yamada M. Carbohydrate mimicry between human ganglioside GM1 and Campylobacter jejuni lipooligosaccharide causes Guillain-Barre syndrome. Proc Natl Acad Sci U S A. 2004; 101:11404-9. [PubMed: 15277677]

24. Kim SC, Tonkonogy SL, Albright CA, Tsang J, Balish EJ, Braun J, Huycke MM, Sartor RB. Variable phenotypes of enterocolitis in interleukin 10-deficient mice monoassociated with two different commensal bacteria. Gastroenterology. 2005; 128:891-906. [PubMed: 15825073]

25. Chou RC, Kim ND, Sadik CD, Seung E, Lan Y, Byrne MH, Haribabu B, Iwakura Y, Luster AD. Lipid-cytokine-chemokine cascade drives neutrophil recruitment in a murine model of inflammatory arthritis. Immunity. 2010; 33:266-78. [PubMed: 20727790]

26. Sieve AN, Meeks KD, Bodhankar S, Lee S, Kolls JK, Simecka JW, Berg RE. A novel IL-17dependent mechanism of cross protection: Respiratory infection with mycoplasma protects against a secondary listeria infection. European Journal of Immunology. 2009; 39:426-438. [PubMed: 19180464]

27. Ye P, Rodriguez FH, Kanaly S, Stocking KL, Schurr J, Schwarzenberger P, Oliver P, Huang W, Zhang P, Zhang J, Shellito JE, Bagby GJ, Nelson S, Charrier K, Peschon JJ, Kolls JK. Requirement of interleukin 17 receptor signaling for lung CXC chemokine and granulocyte colony-stimulating factor expression, neutrophil recruitment, and host defense. J Exp Med. 2001; 194:519-27. [PubMed: 11514607]

28. Xu S, Cao X. Interleukin-17 and its expanding biological functions. Cellular and Molecular Immunology. 2010; 7:164-174. [PubMed: 20383173] 
29. Weichhart T, Costantino G, Poglitsch M, Rosner M, Zeyda M, Stuhlmeier K, Kolbe T, Stulnig T, Horl W, Hengstschlager M. The TSC-mTOR Signaling Pathway Regulates the Innate Inflammatory Response. Immunity. 2008; 29:565-577. [PubMed: 18848473]

30. Moore JE, Barton MD, Blair IS, Corcoran D, Dooley JS, Fanning S, Kempf I, Lastovica AJ, Lowery CJ, Matsuda M, McDowell DA, McMahon A, Millar BC, Rao JR, Rooney PJ, Seal BS, Snelling WJ, Tolba O. The epidemiology of antibiotic resistance in Campylobacter. Microbes Infect. 2006; 8:1955-66. [PubMed: 16716632]

31. Kirkpatrick BD, Tribble DR. Update on human Campylobacter jejuni infections. Curr Opin Gastroenterol. 2010 2010/12/03 ed. 
A
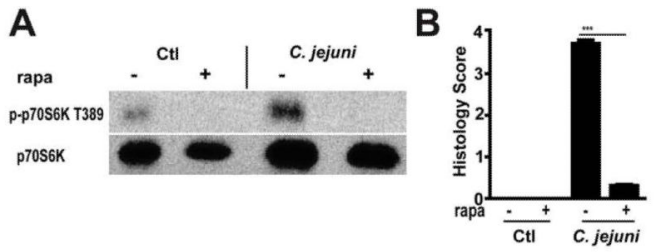

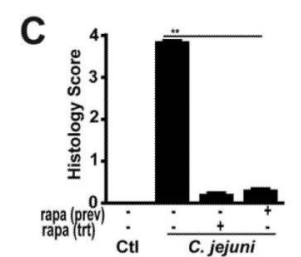

E

E
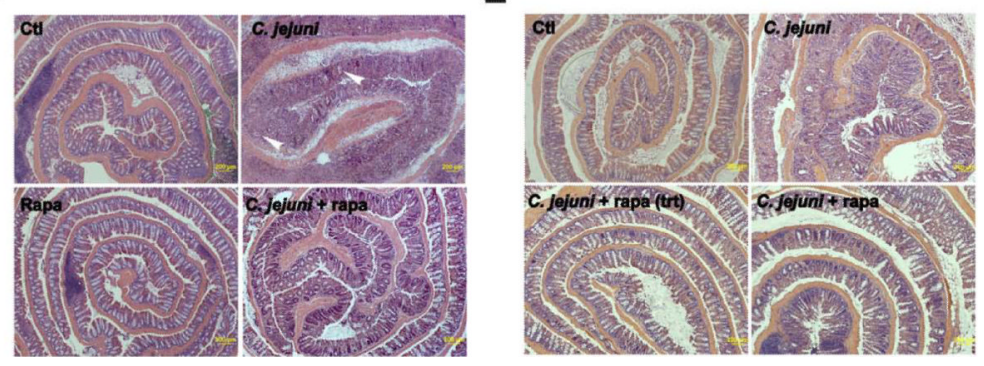

Figure 1. Rapamycin prevents and treats $C$. jejuni induced colitis in $I l 10^{-I-}$; NF- $\mathrm{KB}^{\mathrm{EGFP}}$ mice Cohorts of 5-14 germ-free $I l 10^{-I-}$; NF-kB $\mathrm{KBFP}^{\mathrm{EG}}$ mice were transferred to SPF conditions and immediately gavaged with $10^{9} \mathrm{C}$. jejuni/mouse and then injected i.p. with vehicle control (Ctl, 5\% DMSO), rapamycin (Rapa, $1.5 \mathrm{mg} / \mathrm{kg}$ body weight) for 12 days. For rapamycin treatment (Rapa trt), C. jejuni-infected mice (4 days) were injected i.p. with rapamycin to day 12. Colon was resected, fixed in formaldehyde and sections were stained with H\&E or protein extracts for Western blot analysis. (A) Western blot for total and phosphorylated colonic p70S6. (B) Histological intestinal damage scores of rapamycin prevention on $C$. jejuni infection. (C) Histological intestinal damage score of rapamycin treatment. (D) H\&E representation of the different experimental groups with crypt abscesses (arrow heads). (E) H\&E sections of rapamycin treatment mice. All graphs depict mean \pm SE. **, $\mathrm{P}<0.01, * * *$, $\mathrm{P}<0.001$. Scale bar is $200 \mu \mathrm{m}$. Results are representative of 4 independent experiments. 
A

A

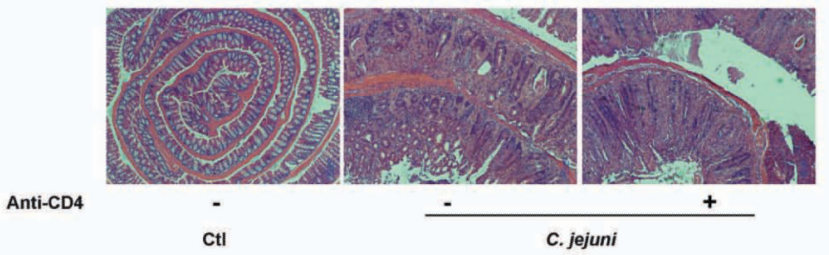

B C

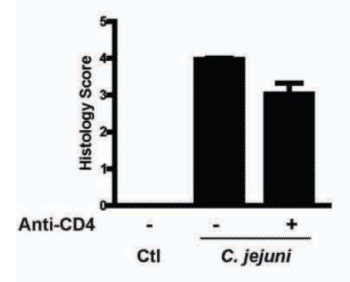

C
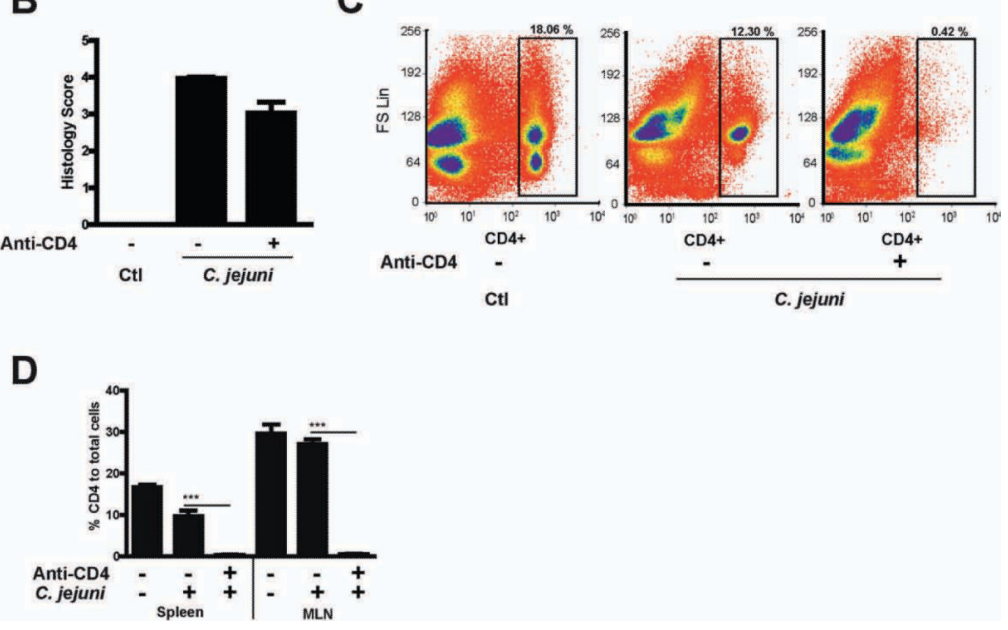

Figure 2. $C$. jejuni induced intestinal inflammation is independent of $\mathrm{CD} 4 \mathrm{~T}$ cells activation Three cohorts of 4-5 germ-free $1 l 10^{-1-}$; NF- $\mathrm{KB}^{\mathrm{EGFP}}$ mice were transferred to SPF conditions and immediately gavaged with $10^{9} \mathrm{C}$. jejuni/mouse and then injected i.p. with vehicle control (Ctl, PBS) or anti-CD4 antibody (anti-CD4, $0.5 \mathrm{mg} / \mathrm{mouse} /$ every 3 days). (A) $\mathrm{H} \& \mathrm{E}$ representation of the different experimental groups. (B) Histological score of intestinal inflammation. (C) Representative flow cytometry analysis of CD4+ cell population change in the spleen. (D) Percentage flow cytometry results of CD4+ cell in the spleen and MLN. All graphs depict mean \pm SE. ***, $\mathrm{P}<0.001$. Scale bar is $200 \mu \mathrm{m}$. Results are representative of 3 independent experiments. 
A

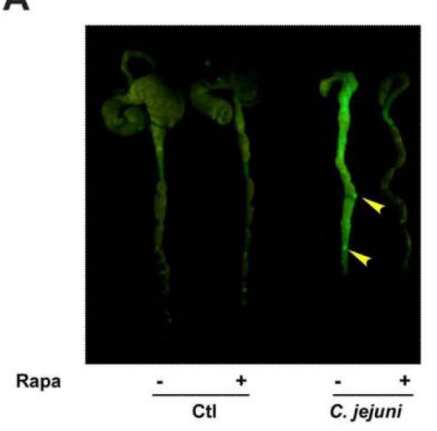

C

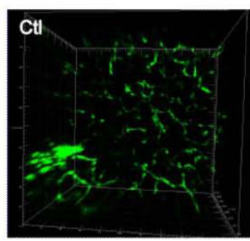

B

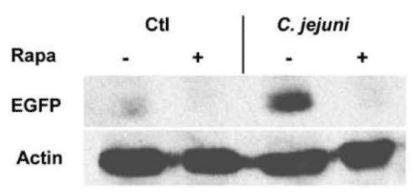

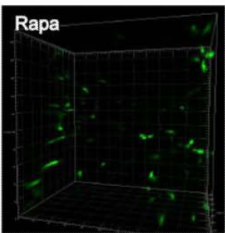
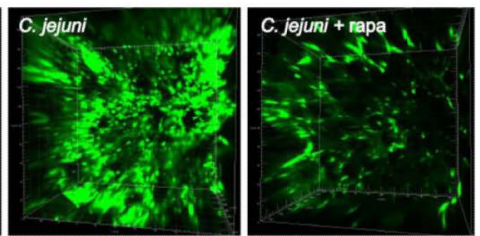

Figure 3. Rapamycin treatment reduces $C$. jejuni induced colonic EGFP expression in $\mathrm{IllO}^{-1-}$; NF-KB ${ }^{\text {EGFP }}$ mice

Four cohorts of 5-14 germ-free $I l 10^{-1-}$; NF- $\mathrm{KB}^{\mathrm{EGFP}}$ mice were transferred to SPF conditions and infected with $C$. jejuni/mice as described in Fig.1. Mice were injected i.p. with vehicle (5\% DMSO) or rapamycin ( $1.5 \mathrm{mg} / \mathrm{kg}$ body weight) for 12 days. (A) EGFP expression in the mouse colon was visualized using a CCD camera macroimaging system. (B) EGFP levels were analyzed by Western blot and (C) confocal microscopy visualization of EGFP positive cells images in 3-D using Imaris. Results are representative of 3 independent experiments. 


\section{A}

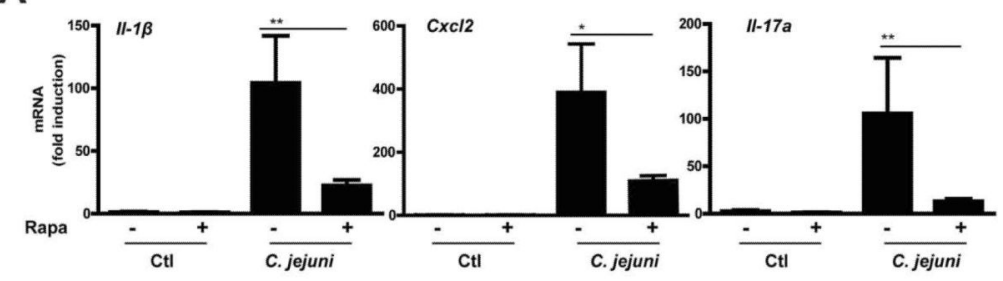

B

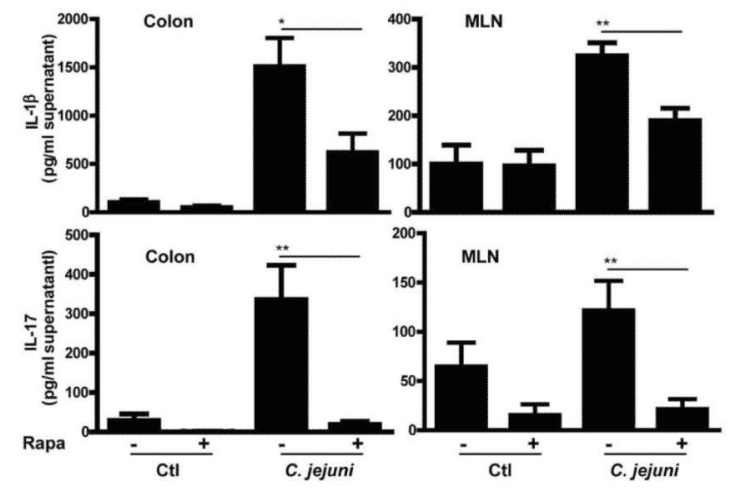

Figure 4. Rapamycin attenuates $C$. jejuni induced expression of proinflammatory mediators Four cohorts of 5-14 germ-free $1 l 10^{-1-}$; NF- $\mathrm{B}^{\mathrm{EGFP}}$ mice were treated as indicated in Fig.1. Colons were resected and RNA extracted using Trizol. (A) Ill $\beta, C x c l 2$ and $I l-17 a$ mRNA accumulation was quantified using an ABI 7900HT Fast Real-Time PCR System and specific primers and data were normalized to Gapdh. (B) Colonic tissues and mesenteric lymph nodes were cultured in RPMI 1640 medium supplemented with $3 \%$ FBS and 1\% antibiotics for $18 \mathrm{hrs}$. Supernatants were collected and IL-1 $\beta$ and IL-17 secretion measured by ELISA. Data represent means $\pm \mathrm{SE} *, \mathrm{P}<0.05^{* *}, \mathrm{P}<0.01$. Results are representative of 3 independent experiments. 

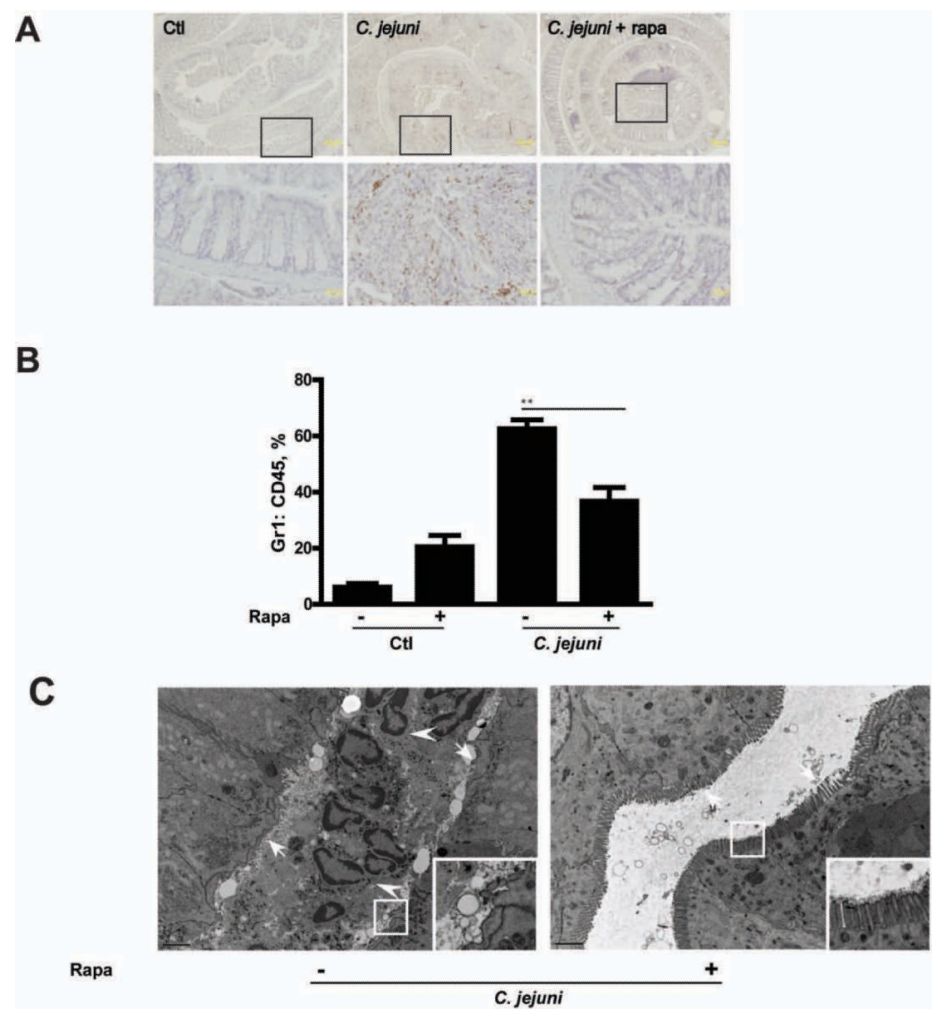

Figure 5. Neutrophils participate in $C$. jejuni induced colitis

Cohorts of 5-7 germ-free $I l 10^{-1-}$; NF- $\mathrm{KB}^{\mathrm{EGFP}}$ mice were transferred to SPF conditions and infected/treated as indicated in Fig.1. (A) IHC representation of MPO positive colonic cells (brown dots) in Control (Ctl), C. jejuni infected, rapamycin (Rapa) treated mice. Lower panels (scale bar $20 \mu \mathrm{m}$ ) are magnified images of area shown in the upper panels (scale bar $200 \mu \mathrm{m}$ ). (B) Flow cytometry analysis for CD $45^{+}$and $\mathrm{Gr}-1^{+}$cells in the peripheral blood of infected and rapamycin-treated mice. (C) TEM images of colonic tissues from C. jejuni infected with/without rapamycin treatment. Left panel shows accumulated neutrophils (arrow heads) in a crypt with microvilli erosion (arrows). Right panel shows absence of neutrophils with intact microvilli (arrows). Inserts in the lower right corners are magnified images of framed area. Scale bar is $2 \mu \mathrm{m}$. Data represent means $\pm \mathrm{SE} * *, \mathrm{P}<0.01$. Results are representative of 3 independent experiments. 
A

Figure 6. mTOR signaling promotes $C$. jejuni invasion of the colon, MLN and spleen Cohorts of 3-4 germ-free $I l 10^{-/-}$; NF- $\mathrm{KB}^{\mathrm{EGFP}}$ mice were transferred to SPF conditions and infected/treated as indicated in Fig.1. (A) Clustered C. jejuni (red dots) in colonic section of infected mice was detected using FISH. Inserts in the lower left corners are magnified images of framed area. Scale bar is $100 \mu \mathrm{m}$. (B) Presence of clustered C. jejuni (red dots) in whole colon tissue was determined using FISH and 3D-imaging. (C) Evidence of intracellular (arrows, left panel) and luminal (right panel) $C$. jejuni in colonic tissues of untreated or rapamycin-treated mice respectively. Lower panels (scale bar $200 \mathrm{~nm}$ ) were magnified images of area shown in the upper panels (scale bar $2 \mu \mathrm{m}$ ). (D) C. jejuni bacterial count in the stool, colon, MLN and spleen of untreated or rapamycin-treated mice. Data represent means $\pm \mathrm{SE} *, \mathrm{P}<0.05$. Results are representative of 3 independent experiments. 


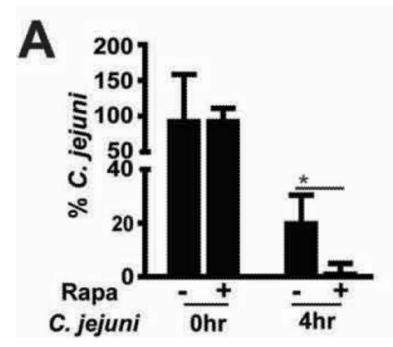

B

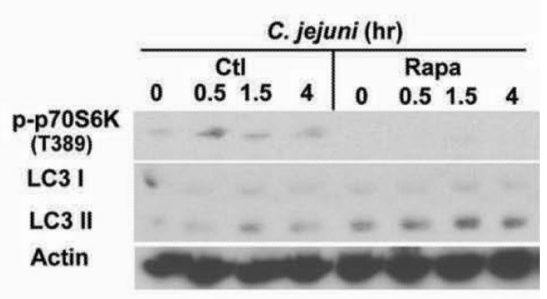

Figure 7. Rapamycin promotes $C$. jejuni eradication and LC3II generation in splenocytes Splenocyte was isolated from $I l 10^{-1-}$ mice and infected with $C$. jejuni (MOI 50) and cultured with rapamycin $(100 \mathrm{nM})$. A gentamycin killing assay at 0 and $4 \mathrm{hr}$ and $\mathrm{WB}$ assay at $0.5,1.5$ and $4 \mathrm{hr}$ were performed. (A) Percentage of $C$. jejuni survival in splenocytes. (B) Western blot of colonic LC3 I/II and phosphorylated p70S6 in infected splenocytes. 